Funda Çorapçíoğlu

M. Memet Özek

Aydin Sav

Deniz Üren

\section{Congenital pineoblastoma and parameningeal rhabdomyosarcoma: concurrent two embryonal tumors in a young infant}

Received: 11 March 2005

Published online: 9 November 2005

(C) Springer-Verlag 2005

F. Çorapçíoğlu

Department of Pediatrics,

Division of Pediatric Oncology,

Kocaeli University,

Kocaeli, Turkey

M. Memet Özek

Division of Pediatric Neurosurgery, Marmara University Medical Center, Istanbul, Turkey

A. Sav

Department of Pathology, Marmara

University Medical Center,

Istanbul, Turkey

D. Üren

BilGen Genetic Diagnosis Center,

Bilkent University,

Ankara, Turkey

\author{
M. Memet Özek ( $\bowtie)$ \\ Acibadem, P.K 195, \\ 34650 İstanbul, Turkey \\ e-mail: mozek@turk.net \\ Tel.: +090-216-4420726
}

\begin{abstract}
Background: Pineoblastomas are very rare brain tumors in fetus and neonates, comprising only $0.9 \%$ of congenital brain tumors. The occurrence of multiple tumors of different histopathologic types in the same individual is a rare event, most often encountered in hereditary cancer syndromes. Case report: We report a female fetus presented with a congenital pineoblastoma at the $32 \mathrm{nd}$ week of gestation, with hydrocephalus and concurrent parameningeal
\end{abstract}

embryonal rhabdomyosarcoma in early infancy. Results: Cytogenetic analysis showed normal karyotype in the peripheral blood of the patient, and p53 mutational analysis revealed no germ line mutations. Discussion: This is the first case with concurrent congenital pineoblastoma and parameningeal embryonal rhabdomyosarcoma in early infancy. We suggest that concurrence of these tumors could be due to mutations in other tumor suppressor genes or secondary to exposure to unknown in utero factors.

Keywords Brain tumor · Congenital · Pineoblastoma · Rhabdomyosarcoma $\cdot$ p53 gene mutation

\section{Introduction}

Congenital neoplasms represent $2.5 \%$ of all tumors in pediatric age $[1,2]$. Although congenital intracranial tumors only account for $0.5-1.5 \%$ of all childhood brain tumors, they are responsible for $5-20 \%$ of the deaths due to neoplasms in this age group [2-4]. Pineoblastomas are uncommon brain tumors in the fetus and neonates, comprising only $0.9 \%$ of congenital brain tumors $[4,5]$. The occurrence of multiple tumors of different histopathologic types in the same individual is even more rare, most often seen in hereditary cancer syndromes. In these syndromes, increased cancer susceptibility results from germ line mutations in various genes controlling the cell growth [6]. We present a case with concurrent congenital pineoblastoma and parameningeal embryonal rhabdomyosarcoma in early infancy. Mutations of a well-defined tumor suppres- sor gene, p53, is studied in the peripheral blood cells of the patient.

\section{Case report}

A 28-year-old woman at the 32nd week of gestation was referred to the Division of Pediatric Neurosurgery at Marmara University Medical Center due to dilated third and lateral ventricles and a hyperechoic lesion in the pineal area detected by fetal ultrasonography (Fig. 1a). The ultrasonography revealed no extracranial abnormalities, and medical history of the mother including pregnancy was uneventful. A fetal MRI was performed on the 33rd week of gestation, which showed a mass lesion $2 \times 1 \times 1 \mathrm{~cm}$ in the pineal gland region causing hydrocephalus (Fig. 1b). Family history was negative for malignancies, and there were no 
Fig. 1 Prenatal ultrasonography and MRI. a Fetal ultrasonography revealed dilated ventricules and a hyperechoic lesion in the pineal area (arrow). b Axial T2 weighted fetal MR image demonstrates a cystic mass lesion with heterogeneous signal intensity in pineal region with colpocephaly (arrow)
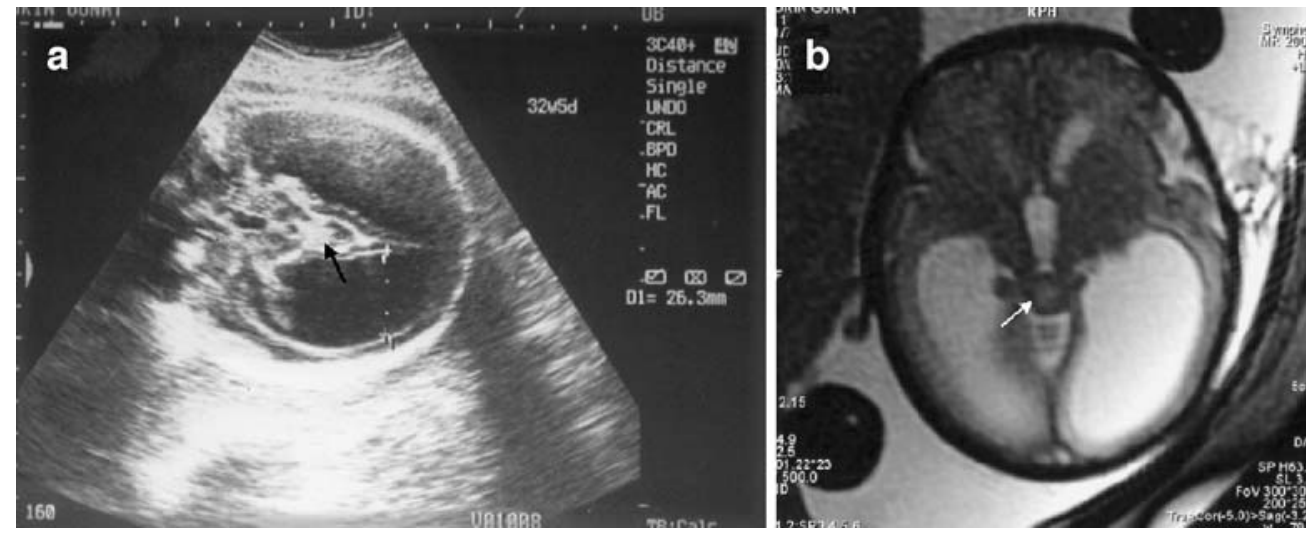

predisposing hereditary disorders related to brain tumors. No in utero exposure to environmental risk factors could be identified from the history. Due to progressive increase in ventricule dimensions, at the 35th week of gestation, the mother underwent cesarean section. The female newborn was in good condition and had a birth weight of 2,800 g. There was no congenital anomalies, and physical examination was normal, except for macrocephaly and wide bulging fontanels and dilated scalp veins. On the MRI of the first postnatal day, the size of the pineal gland mass was increased to $3.5 \times 1.5 \times 2 \mathrm{~cm}$, as well as the ventricular size (Fig. 2a). On the postnatal first day, a ventriculoperitoneal shunt was installed to relieve the increased intracranial pressure and wait for clinical stability. Alpha fetoprotein levels of cerebrospinal fluid and serum obtained during the operation were 3,199 and 91,000 IU/ml, respectively. The general condition of the baby was quite well. Although hydrocephalus was decompressed, on postnatal 45th day, MRI revealed prominent progression of the mass lesion (Fig. 2b). On the postnatal 47 th day, radical surgery was performed using the right occipital transtentorial approach. A mass with cystic and solid components was seen at the location of the pineal region. The mass was adherent to both the internal cerebral veins and the vein of Galen. The mass was also extending into the posterior fossa, compressing the cerebellum with an ependymal infiltration. After aspiration of xanthochromic cyst fluid, the fragile solid portion of the tumor was completely removed with the help of ultrasonic aspirator. The postoperative period was uneventful. MRI on the postoperative first day and postnatal 30th day showed no evidence of residual mass (Fig. 2c). The MRI of the postoperative tenth day presented normal-sized ventricles (Fig. 2d). Cerebrospinal fluid cytologic examination and whole-spinal MRI revealed no drop metastasis or meningeal dissemination. Histopathologically, excised tumoral mass showed a diffuse, infiltrative, cellular tumor composed of small round cells with hyperchromatic nuclei and scant amphophilic cytoplasm. Tumor had cellular atypia but low mitotic index (0-1/hpf) and lacked necrosis and vascular endothelial proliferation. Immunohistochemically, tumor cells were reactive for vimentin and synaptophysin. S100 and glial fibrillary acidic protein (GFAP) did not show any immunoreactivity. MIB-1 proliferative index was $33 \%$. The final histopathologic diagnosis was pineoblastoma (Fig. 3).

The patient was started on The Societé Française Oncologie Pédiatrique (SFOP) chemotherapy protocol on the postoperative tenth day [7]. She received two courses of chemotherapy with 21 days interval. Original regimen was: course 1; carboplatin $15 \mathrm{mg} / \mathrm{kg}$ on day 1, procarbazine $4 \mathrm{mg} /$ $\mathrm{kg}$ on days $1-7$ and course 2; etoposide $5 \mathrm{mg} / \mathrm{kg}$ on days 1 and 2 , cisplatinum $1 \mathrm{mg} / \mathrm{kg}$ on days 1 and 2 . All drugs were used with a dose reduction schedule due to the young age of the patient. After the second course of the chemotherapy, she was admitted to the hospital center with fascial asymmetry. A solid painless mass was palpated on the right maxillofascial region. MRI showed a mass lesion originating from right maxillary sinus, in addition to liver, lung, and vertebral metastasis (Fig. 4a,b). An incisional biopsy of the maxillary mass was performed at the age of 3 months, and histologically, tumor consisted of densely packed small round cells with intervening hypocellular loose stroma. Tumor cells were mostly hyperchromatic small round cells with occasional spindle cells and few number of cells having eosinophilic cytoplasm. Immunohistochemically, tumor cells were diffusely positive with vimentin and negative for leukocyte common antigen (LCA), cytokeratin, neuron-specific enolase (NSE), chromogranin, and S-100. Desmin was detected in the cells with discernible cytoplasm (Fig. 5). The pathologic diagnosis was embryonal rhabdomyosarcoma. Cytogenetic analysis was done from the peripheral blood lymphocyte cultures that showed normal karyotype. The p53 gene mutation was studied in peripheral blood cells of the patient to identify any genetic etiology and to give genetic counseling. We sequenced exons 2 thru 11 including the splice sites to search for mutations in the genomic DNA extracted from peripheral blood cells of the patient. The primer sequences were: ex2F (ccagggttggaagtgtctcat), ex3R (gag cagtcagaggaccaggtc), ex4F (gacctggtcctctgactgct), ex4R (gccaggcattgaagtctcat), ex5F (acttgtgccetgacttcaact), ex6R (gccactgacaaccacctta), ex7F (cctcatcttgggcctgtgtt), ex7R (tggaagaaatcggtaagaggtg), ex8F (ggagtagatggagcctggttt), ex9R (aagaaaacggcatttgagtg), ex10F (caattgtaacttgaaccatc), 
Fig. 2 a T1 weighted contrast image shows that the pineal mass was enlarged and reached approximately $3 \times 1.5 \times 2 \mathrm{~cm}$ dimensions (arrow). The cystic components of the lesion are better demonstrated, and hydrocephalus is more prominent. b On postnatal 45th day, axial T1 weighted MR image after i.v. contrast shows decrease in ventricular size due to shunt placement. Further enlargement of the pineal mass is seen (arrow). c and $\mathbf{d}$ After successful surgery, the mass lesion was removed totally. There is no evidence of residual tumor on early (c) and late (d) postoperative T1 weighted contrast enhanced axial MR images
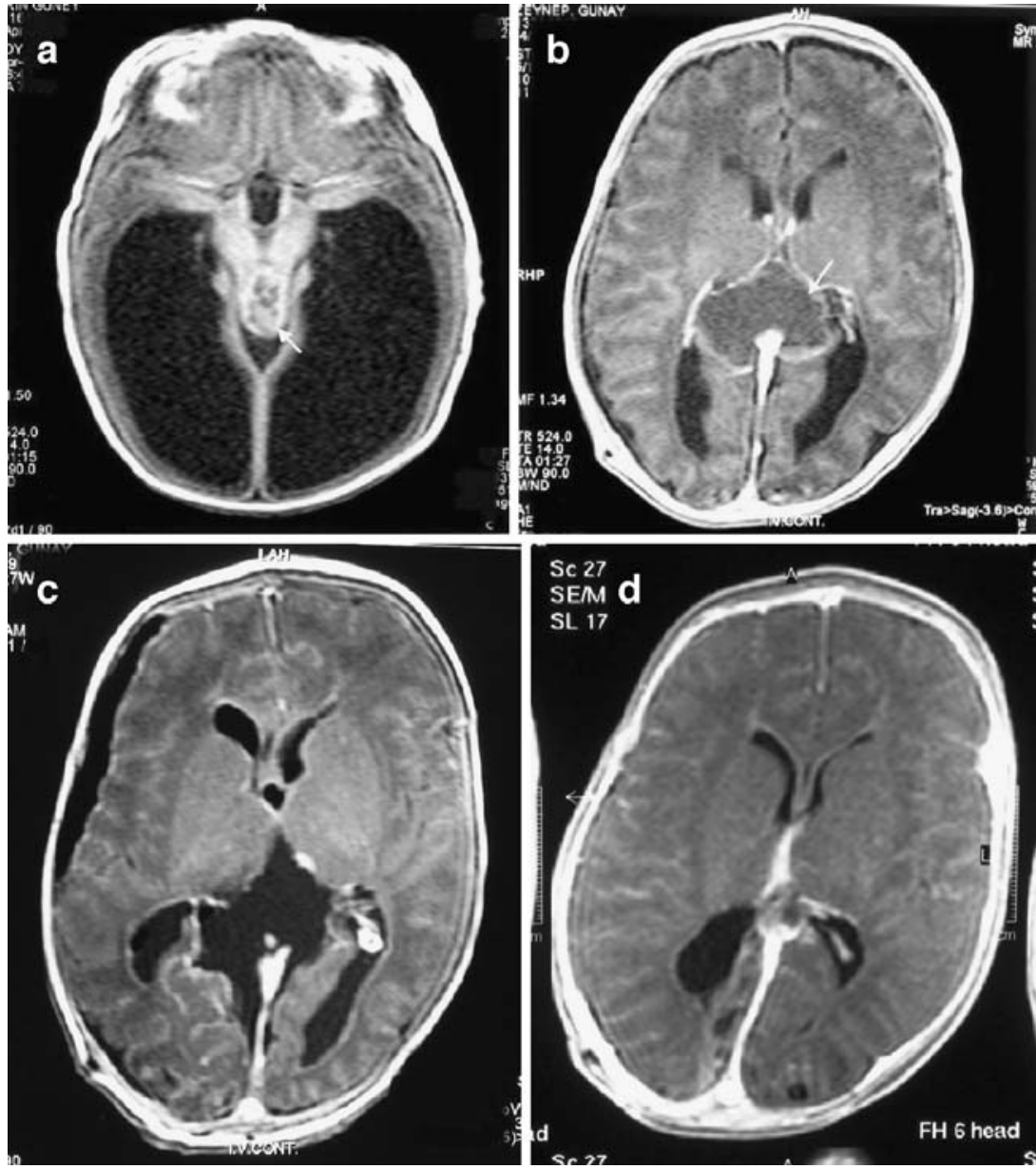

Fig. 3 a Tumor composed of small round cells infiltrating the neural tissue $(\mathrm{H} \& \mathrm{E}, \times 100)$. b Diffuse strong synaptophysin immunopositivity in tumor cells (streptavidin-biotin, $\times 200$ ). c Diffuse strong vimentin immunopositivity in tumor cells (streptavidin-biotin $\times 200$ )

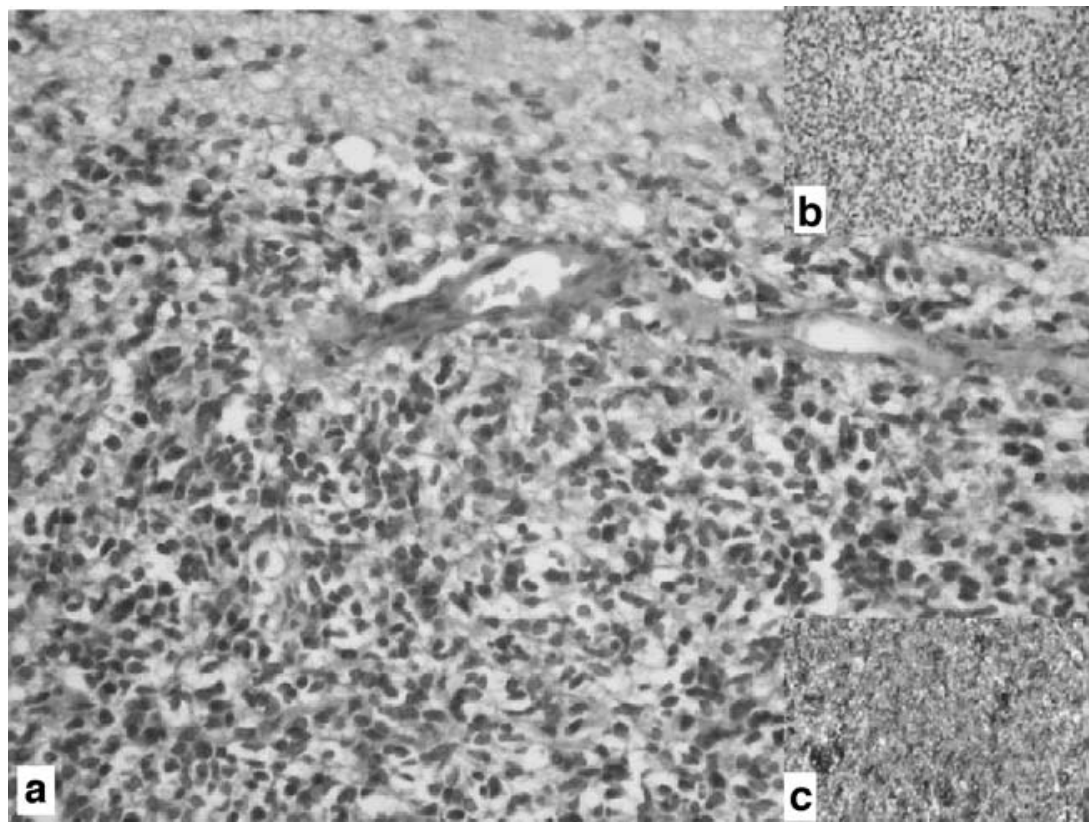


ex10R (gggtttggatgttctgtgga), ex11F (gcacagaccctctcactcatgt), ex11R (tcccaaacatccctcacagt). The amplification conditions were as follows: $95^{\circ} \mathrm{C} 2 \mathrm{~min}$ initial denaturation, followed by 35 cycles of $94^{\circ} \mathrm{C} 1 \mathrm{~min}, 60^{\circ} \mathrm{C} 1 \mathrm{~min}$, and $72^{\circ} \mathrm{C}$ $1 \mathrm{~min}$, except exon 10 , for which the annealing was $56^{\circ} \mathrm{C}$. After amplification, the PCR products were purified by QIAquick PCR purification kit (Qiagen) and the cycle sequencing reaction was done using the Big Dye Terminator Cycle Sequencing Kit (Applied Biosystems). The sequencing analysis was done on the Perkin Elmer-310 Genetic Analyzer.

There was no disease-causing mutation or other alterations, except that the patient carried the $\arg 72$ variant at codon 72 of exon 4 , as homozygous.
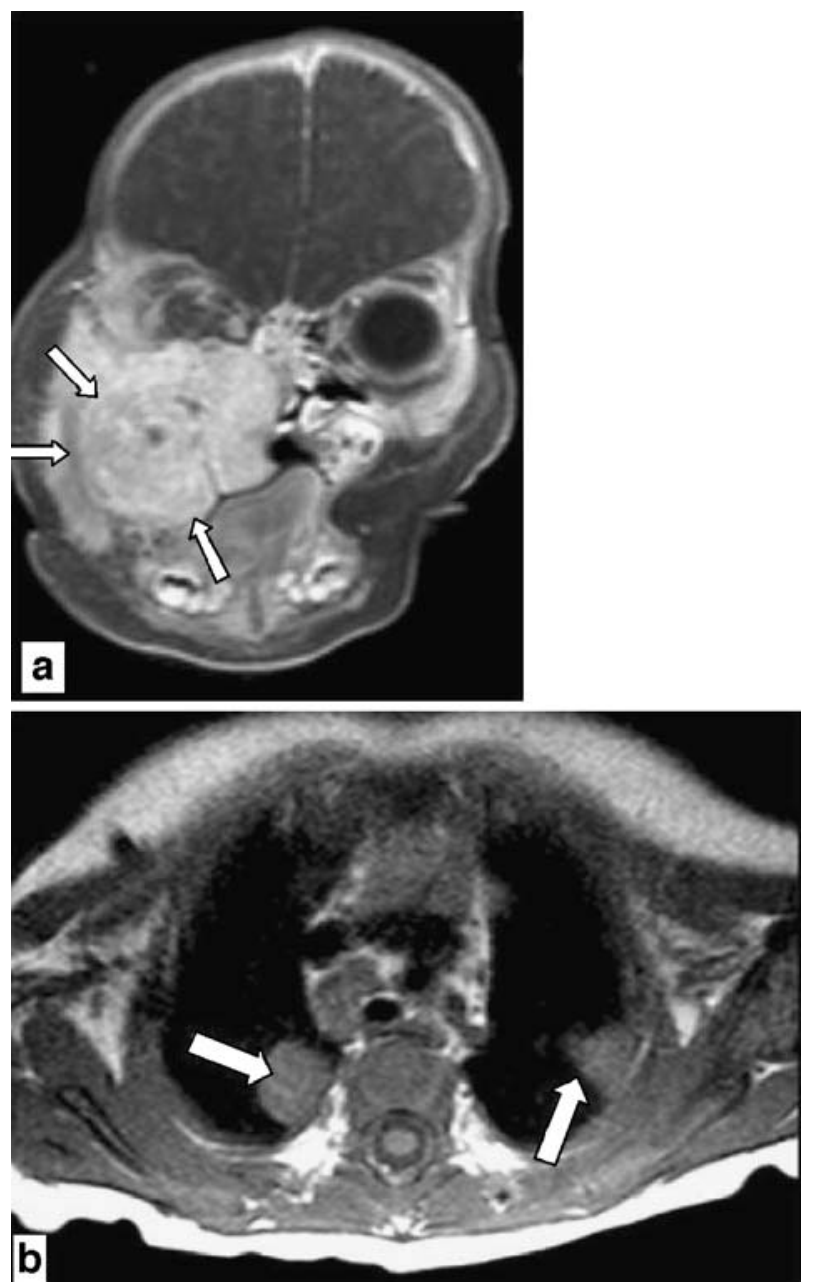

Fig. 4 a Coronal fat saturated T1 weighted MR image demonstrate a mass lesion (arrows) on the right side originating from pterygoid muscles, obliterating the maxillary sinus, invading the inferior wall of the orbit, obliterating the fatty plane under inferior rectus muscle, invading inferior and middle conchae, reaching the nasal septum, eroding the palatum durum. The globe is displaced superiorly by the mass effect. b Peripheral, round metastatic lung lesions are seen on axial T1 weighted image (arrows)
After informing the family about the poor prognosis of the tumors, the parents agreed to proceed with chemotherapy to stop progression of the maxillary mass without any further invasive intervention. Three courses of EVAC chemotherapy regimen were administered (etoposide $5 \mathrm{mg} / \mathrm{kg}$ on days 1 and 2 , vincristine $0.05 \mathrm{mg} / \mathrm{kg}$ on day 1 , actinomycin-D 15 gamma $/ \mathrm{kg}$ on days $1-3$, cyclophosphamide $60 \mathrm{mg} / \mathrm{kg}$ on day 1; all drugs were used with a dose reduction due to the young age of the patient). After the two courses of EVAC chemotherapy protocol, maxillofascial mass showed clinical regression and abdominal ultrasonography revealed complete regression of liver metastases. Following the third EVAC chemotherapy course, the patient presented with convulsions and recurrence of pineoblastoma was seen on cranial MRI (Fig. 6a,b). The family refused no further chemotherapy, and the patient died at home in the following days.

\section{Discussion}

The use of perinatal ultrasonography and fetal MRI permit early detection of congenital brain tumors [8]. The initial ultrasound finding in our case was ventricular dilatation and suspicious hyperechoic lesion in the pineal region. Fetal intracranial tumors rarely cause in utero ventricular dilatation, with a prevalence of only $1.1 \%$ of all the patients with in utero hydrocephalus $[9,10]$. In the present case, the hyperechoic pineal lesion in the fetal ultrasonography led us to a fetal MRI that confirmed a tumoral etiology for the obstructive hydrocephalus. Fetal MRI is a more accurate imaging method in depicting the tumor and its characteristics as well as in taking a decision with regard to management of pregnancy $[8,9]$. If such a tumor is detected before the 24th week of gestation, termination of pregnancy could be discussed, while the prognosis of the fetus has been generally poor [3]. Mortality during delivery was reported to be $92 \%$ in infants with congenital brain tumors [8]. Early intervention for shunting procedure and successful total resection of the brain tumor provided a good early neurological outcome.

Congenital central nervous system tumors are rare and their location, histologic types, biologic behavior and response to therapy are different from those in the older children [2,4]. Primary intracranial tumors are mostly located in the supratentorial region in neonates and young infants, in contrast to older children in whom the majority are found within the posterior fossa $[2,11,12]$.

Primitive neuroectodermal tumors (PNETs) including pineoblastomas have been noted to have the worst prognosis, metastasizing widely throughout the cerebrospinal fluid pathways, invading the meninges and the spinal cord $[4,7]$. Outcome is related to the size, location, surgical resectability, and the condition of the infant at the time of diagnosis [4]. Surgical resection is an important component of the multimodal therapy. Due to low prevalence of pineoblastomas and 
Fig. 5 a Pleomorphic tumor cells with round and spindle morphology, mixed with larger cells with cytoplasm and loose myxoid stroma (H \& E, ×200) b Cytoplasmic desmin immunoreactivity within the tumor cells (streptavidin-biotin, $\times 400$ )

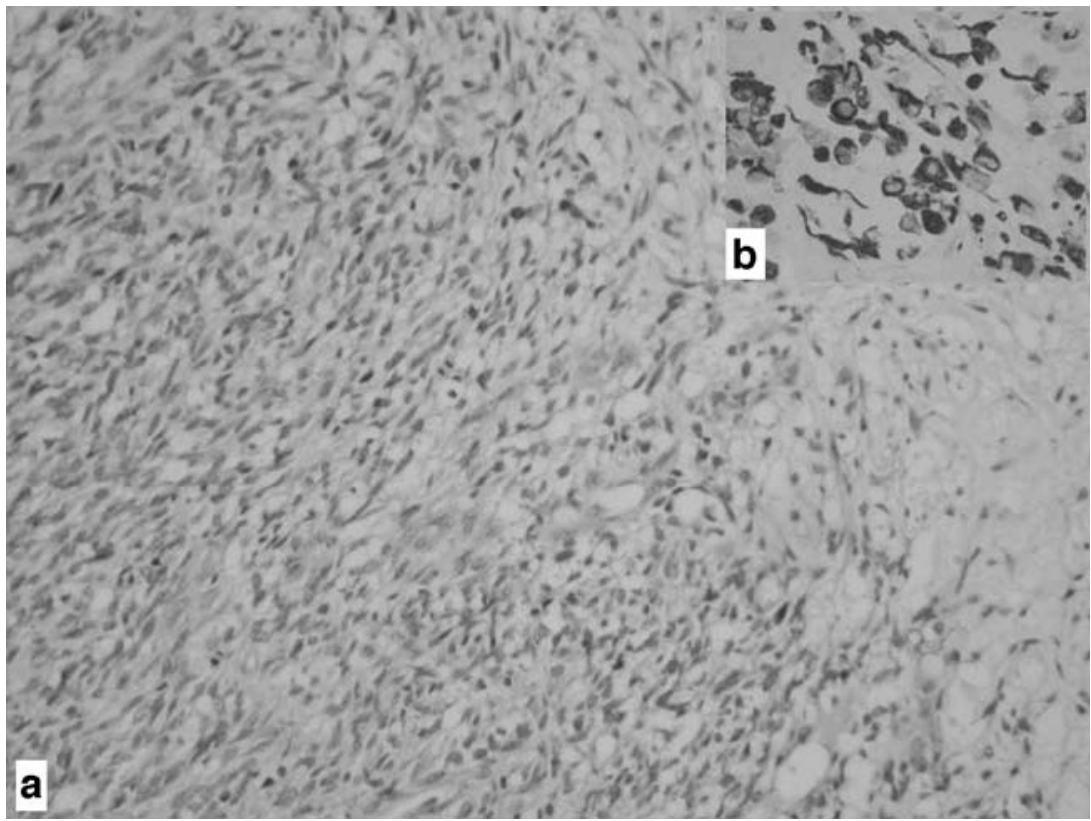

operative risks in this age group, it has been quite difficult to determine the results of surgical removal of the congenital brain tumors in the newborns and infants [7, 8]. In our case, a ventriculoperitoneal shunt was inserted on the first postnatal day and tumor could be resected without any surgical complication within the neonatal period.

Although cranial and spinal radiotherapy following surgery is the gold standard for the treatment of pineoblastoma, with its severe and irreversible sequelae, it is not a recommended therapeutic option in infancy $[5,11]$. Chemotherapy alone, at least in conventional doses, appears to be insufficient as treatment for younger children with pineoblastomas, where rapid tumor progression and death is almost unavoidable [5]. Marec-Berard et al. [7] reported 25 children under 5 years of age with supratentorial PNETs treated with BB SFOP protocol. Postoperative chemotherapy alone maintained 29 and $14 \%$ overall survival at 2 and 5 years, respectively; relapse-free survival was $4 \%$ at 2 years [7]. In the presented case, a chemotherapy directed to rhab- domyosarcoma was administered following two courses of chemotherapy administered for pineoblastoma. Cessation of the chemotherapy directed to pineoblastoma may be the reason for early recurrence of the brain tumor.

Although PNETs may metastasize to distant sites, such as the lungs, liver, lymph nodes, and bone marrow, in our patient, distant metastatic lesions were most likely to originate from rhabdomyosarcoma because primary brain tumor was already in remission at the time, and these were common metastatic sites for rhabdomyosarcoma. Rhabdomyosarcoma is the most common soft tissue sarcoma of the childhood [13]. It is known that the risk of developing two or three primary neoplasms is higher in patients with soft tissue sarcoma compared with the general cancer population [14].

Multiple tumors of different histopathologic types in the same individual is rare, generally seen in hereditary cancer syndromes. In these syndromes, increased cancer susceptibility results from germ line mutations in various genes controlling cell growth $[6,13,15]$. Although there was no family
Fig. 6 a Axial image shows the reduced dimensions of rhabdomyosarcoma (black arrow).

However, reoccurrence of pineablastoma is seen (white arrow). b. Sagittal T1 weighted image demonstrates the recurrent mass lesion on the pineal gland region, filling the fourth ventricle anteriorly and the superior cerebellar cistern posteriorly. Cerebellar tonsils are herniated $10 \mathrm{~mm}$ through foramen magnum
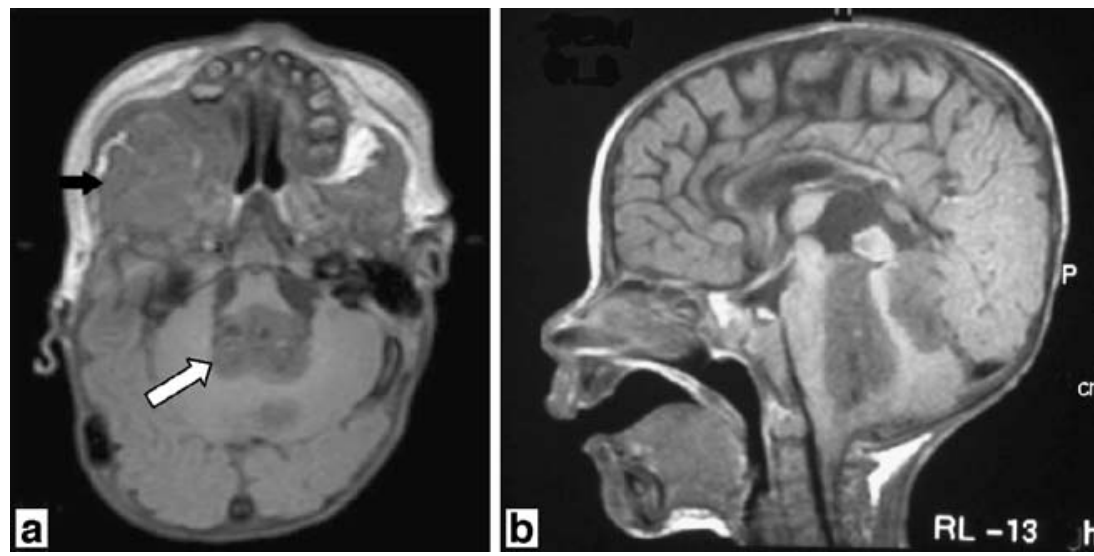
history of cancer, a genetic susceptibility could have been a causative factor in the development of two different tumors.

The $\mathrm{p} 53$ tumor suppressor gene, located on the short arm of human chromosome 17, encodes a 53-kDa phosphoprotein that functions as a regulator of cell proliferation and apoptosis. Alterations of the $\mathrm{p} 53$ gene or its encoded protein are the most common genetic abnormalities observed in human cancers, having been associated with virtually every sporadically occurring malignancy [13]. Even if the family history was negative for any other cancer consistent with the Li-Fraumeni Syndrome (LFS), germ line p53 mutation must be kept in mind in children with multiple primary tumors [15]. Majority of the patients with rhabdomyosarcoma are genetically susceptible to tumor development. Rhabdomyosarcoma is the most common sarcoma described in LFS and is associated with a carrier state of a constitutionally altered allele of the p53 tumor suppressor gene [13-15]. Diller et al. [13] reported that the presence of p53 mutation might predispose children to the development of rhabdomyosarcoma at an early age. Children with astrocytoma and oligoastrocytoma most commonly have inherited p53 gene mutations. In contrast, p53 gene mutation has been rare in the tumorigenesis of pineoblastoma $[16,17]$.

Concurrent combination of pineoblastoma and rhabdomyosarcoma has not been reported before. The presented case has no family history of cancer and has no detectable mutation for p53 tumor suppressor gene carried as germ line. We suggest that concurrence of these tumors could be due to mutations in other tumor suppressor genes or to exposure of unknown in utero factors.

\section{References}

1. Balestrini MR, Micheli R, Giordano L, Lasio G, Giombini S (1994) Brain tumors with symptomatic onset in the first two years of life. Childs Nerv Syst 10:104-110

2. Isaacs HJR (2002) I. Perinatal brain tumors: a review of 250 cases. Pediatr Neurol 27:249-261

3. Rickert CH, Porobst-Cousin S, Louwen F, Feldt B, Gullotta F (1997) Congenital immature teratoma of the fetal brain. Childs Nerv Syst 13:556-559

4. Isaacs HJR (2002) II. Perinatal brain tumors: a review of 250 cases. Pediatr Neurol 27:333-342

5. Jakacki RI (1999) Pineal and nonpineal supratentorial primitive neuroectodermal tumors. Childs Nerv Syst 15:586-591

6. Brockmeyer DL, Walker ML, Thompson G, Fults DW (1997) Astrocytoma and pineoblastoma arising sequentially in the fourth ventricle of the same patient. Case report and molecular analysis. Pediatr Neurosurg 26:36-40

7. Marec-Berard P, Jouvet A, Thiesse P, Kalifa C, Doz F, Frappaz D (2002) Supratentorial embryonal tumors in children under 5 years of age: an SFOP study of treatment with postoperative chemotherapy alone. Med Pediatr Oncol 38:83-90
8. Im SH, Wang KC, Kim SK, Lee YH, Chi JG, Cho BK (2003) Congenital intracranial teratoma. Prenatal diagnosis and postnatal successful resection. Med Pediatr Oncol 40:57-61

9. Mazouni C, Porcu-Buisson G, Girard N, Sakr R, Figarella-Ballanger D, Guidicelli B, Bonnier P, Gamerre M (2003) Intrauterine brain teratoma: a case report of imaging (US, MRI) with neuropathologic correlations. Prenat Diagn 23:104-107

10. Girard N, Raybaud C, Gambarelli D, Figarella-Ballanger D (2001) Pediatric MR neuroimaging. Fetal brain MR imaging. Magn Reson Imaging Clin North Am 9: $19-56$

11. Cho BK, Wang KC, Man DH, Jung HW, Kim HJ, Han DH, Choi KS (1998) Pineal tumors: experience with 48 cases over 10 years. Childs Nerv Syst 14:53-58

12. Oi S, Matsuzawa K, Choi JU, Kim DS, Kang JK, Cho BK (1998) Identical characteristics of the patient populations with pineal region tumors in Japan and in Korea and therapeutic modalities. Childs Nerv Syst 14:36-40
13. Diller L, Sexsmith E, Gottlieb A, Li FP, Malkin D (1995) Germline p53 mutations are frequently detected in young children with rhabdomyosarcoma. J Clin Invest 95:16061611

14. Merimsky O, Kollender Y, Issakov J, Bickels J, Flusser G, Gutman M, Lev-Chelouche D, Inbar M, Meller I (2001) Multiple primary malignancies in association with soft tissue sarcomas. Cancer 91:1361-1371

15. Khayat CM, Johnston DL (2004) Rhabdomyosarcoma, osteosarcoma, and adrenocortical carcinoma in a child with germline p53 mutation. Pediatr Blood Cancer 43:683-686

16. Stander M, Peraud A, Leroch B, Kreth FW (2004) Prognostic impact of TP53 mutation status for adult patients with supratentorial World Health Organization grade II astrocytoma or oligoastrocytoma. A long-term analysis. Cancer 101: 1028-1035

17. Tsumanuma I, Sato M, Okazaki H, Tanaka R, Washiyama K, Kawasaki T, Kumanishi T (1995) The analysis of p53 tumor suppressor gene in pineal parenchymal tumors. Noshuyo Byori 12:39-43 